\title{
Entre vários campos: a obra de Fábio Moon e Gabriel Bá e a literatura brasileira
}

\author{
Marcel Vejmelka*
}

\section{HQ e a literatura}

A obra dos autores e ilustradores brasileiros Fábio Moon e Gabriel Bá, presente e reconhecida tanto no Brasil quanto a nível internacional, evidencia de forma particular os conflitos e as tensões existentes entre os diferentes campos envolvidos na produção e circulação de histórias em quadrinhos. As observações aqui apresentadas se situam dentro da proposta teórica e metodológica da sociologia da literatura, iniciada por Pierre Bourdieu, que caracteriza os campos de produção cultural, entre eles especificamente os campos literários, como marcados por uma polaridade fundamental entre o estético e o comercial. O interessante dessa proposta é a possibilidade de enxergar essas duas forças como simultâneas e não necessariamente exclusivas num dado momento em determinado campo:

On peut rendre compte de la structure chiasmatique de cet espace, dans lequel la hiérarchie selon le profit commercial (théâtre, roman, poésie) coexiste avec une hiérarchie de sens inverse selon le prestige (poésie, roman, théâtre), pour un modèle simple prenant en compte deux principes de différenciation (BourdiEu, 1992, p. 167).

É esta contradição, somente aparente, que inspira a observar a história e evolução da produção de HQ, por parte dos gêmeos Moon e Bá, num jogo de forças e dinâmicas parcialmente conflitivas, aparentemente contraditórias, mas que afinal compõem um panorama concludente e interessante das caraterísticas desse gênero e dos seus respectivos campos nacionais e internacionais. Num primeiro momento, parece evidente que Moon e Bá figuram no âmbito das HQ: a sua obra publicada no Brasil e em outros países constitui já uma vasta bibliografia, e eles são considerados os representantes mais eminentes das HQ brasileiras em nível nacional e internacional. Mas não é tão evidente o que isto significa e implica com respeito às dinâmicas das quais a sua obra forma parte.

Doutor em Estudos Latino-americanos/Brasileiros pela Universidade Livre de Berlim, professor do Departamento de Espanhol e Português, Faculdade o6 Tradução, Linguística e Estudos Culturais da Universidade Johannes Gutenberg de Mainz, campus de Germersheim. E-mail: vejmelka@uni-mainz.de. 
A questão fica mais complicada e interessante ao perguntarmos se as HQs fazem ou não fazem parte do campo literário. Essa discussão é fundamental e infinita, e não é minha intenção continuá-la aqui. A perspectiva dos campos culturais e literários evidencia uma complexidade particular no caso das HQs: em primeiro lugar é preciso reconhecer a discussão histórica a respeito da inclusão ou não das HQs no âmbito da literatura em sentido tradicional. Dependendo do ponto de vista ou do posicionamento do estudioso, as HQs formam parte da literatura ou representam um campo separado, próprio. Isso fica bem visível numa afirmação de Marie-Manuelle da Silva, referindo-se às reflexões de Jan Baetens:

Nesse âmbito, propomos examinar alguns aspectos relativos à constituição de um campo, a "banda desenhada literária" ou "literatura desenhada" (BAETENS, 2009), focando a questão "tradicional” da adaptação dos clássicos da literatura francesa, mas também problemáticas formais relativas a narrativas visuais consideradas como uma nova forma de literatura (SILVA, 2012, p. 80-81).

A questão se decide, portanto, entre uma visão "tradicional" das HQs enquanto uma forma específica da literatura e uma visão mais "autônoma", vendo a HQ enquanto forma própria de produção cultural. Na sua tese de doutoramento, Lucas Piter Costa exemplifica este conflito com o subgênero do romance gráfico, originário do campo literário norte-americano enquanto graphic novel, optando por uma visão menos restrita e mais autônoma da HQ:

O peso da palavra novel (e suas traduções, nem sempre felizes: novela, em espanhol; romance e novela em português; roman, em francês), outrora restrito à Literatura, faz pensar que essa instituição se renova, ampliando sua linguagem com uma nova forma de romance, quando, na verdade, é a própria concepção de romance como objeto restrito da Literatura que está em jogo (CosTA, 2016, p. 77).

Assim, Costa confirma um posicionamento bem estabelecido hoje em dia, de que não se pode reduzir a HQ à condição de literatura, subsumindo-a ou até apropriando-a para esse campo. Nessa perspectiva, ela constitui um campo próprio, com ligações variadas com outros campos, além do literário, principalmente com as artes gráficas e visuais, também com o audiovisual, como aponta Santiago García no seu estudo abrangente do romance gráfico enquanto gênero:

The phenomenon affects not only the literary world, but the world of visual art as well. Comics expositions are no longer quaint events, but increasingly gain access to the spaces of high culture based on their own merits, and not as footnotes to "true art" (GARCÍA, 2015, p. 8).

Mais ainda, segundo Baetens e Sánchez-Mesa (2017, p. 9), as HQs se situam no centro das dinâmicas da produção artística "transmidiática". Porém, compartilham 
com a literatura uma parte do campo, e com isso, parte da sua sistematicidade e de seus mecanismos básicos. Com esta devida cautela, portanto, as HQs podem ser vistas e analisadas enquanto literatura, formando parte do campo literário. $\mathrm{Na}$ perspectiva de uma vertente ampliada e dinamizada dos estudos literários, o que pode ser observado de forma produtiva é identidade de muitos atores e mecanismos de produção, publicação, divulgação e recepção entre HQ e literatura em sentido clássico: de editoras, distribuidoras, livrarias, do suporte impresso, da dimensão narrativa etc. ${ }^{1}$ Esse "território compartilhado" permite analisar as HQs a partir de uma perspectiva originada nos estudos da literatura, mas sempre como visitantes na interdisciplinaridade dos comic studies (cf. ETTER; STEIN, 2016).

Retomando a perspectiva dos estudos literários em sentido tradicional, as HQs ainda são consideradas "subliteratura" pelo sistema literário estabelecido e pela academia tradicional, como confirma Klaus Kaindl: "As HQs tem que lutar muito para se impor no 'campo de batalha' do campo literário - em términos de Bourdieu - como também enquanto objeto no campo acadêmico" (KAINDL, 2010, p. 332). ${ }^{2}$ Por outro lado, as HQ estão vivendo uma fase de crescente êxito comercial e simbólico internacional, diversificando a sua produção entre gibis e graphic novels, revendo a sua própria história e tradição, construindo cânones próprios e intensificando a sua diferenciação interna com subgêneros ou subcampos particulares, como mostra Monika Schmitz-Emans:

\section{PÃEZINHOS}

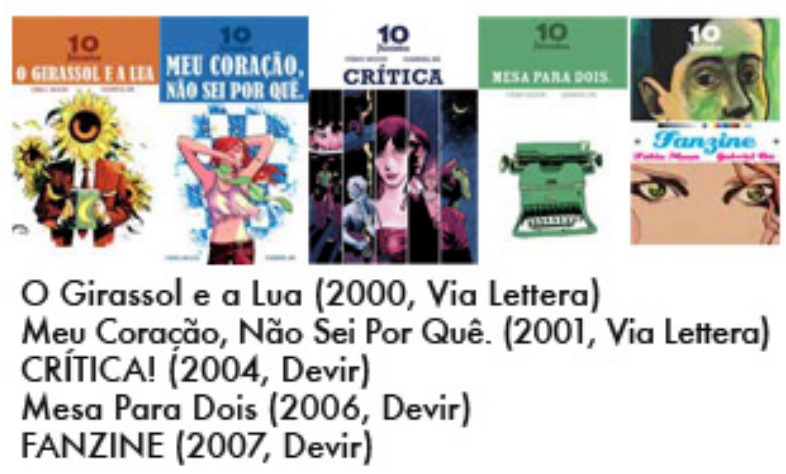

FIGURA 1 - http://www2.uol.com.br/1opaezinhos/images/index-1opaezinhos.jpg.

\footnotetext{
Bourdieu, ao estudar a "autonomia relativa" do campo editorial, nos dá uma orientação fundamental a respeito de como visualizar a diferenciação entre o campo editorial literário "clássico" e o campo editorial das HQ: "Il faut prendre pour objet le champ editorial comme espace social relativement autonome - c'est-à-dire capable de retraduire selon sa logique propre toutes les forces externes, économiques et politiques notamment -, dans lequel les stratégies editoriales trouvent leur principe" (BOURDIEU, 1999, p. 6).

2 "Comics hatten es sehr schwer, sich sowohl auf dem, um es mit Bourdieu zu formulieren, ,Kampfplatz' des literarischen Felds als auch - als Gegenstand - auf dem wissenschaftlichen Feld zu behaupten".
} 
Due to the high artistic quality of many recent graphic novels, there is a remarkable tendency to create and confirm a canon-a process that might be compared at least tentatively to the post-colonial canonization process of non-Western 'literatures' after their having been ignored in the past-or to engage in processes of canonization concerning marginalized forms of literary writing (such as women's literature) (ScHMITZEMANS, 2013, p. 391). ${ }^{3}$.

Particularmente interessante é, nesse caso, o âmbito fronteiriço entre os dois subcampos das HQs, onde uma obra pode funcionar como HQ e como "literatura" ao mesmo tempo. Grande parte da obra dos gêmeos Fábio Moon e Gabriel Bá me parece situar-se justamente nessa fronteira, seja enquanto romance gráfico, seja enquanto HQ com elevada ambição narrativa e estética. O primeiro exemplo disso é a série 10 pãezinhos - com as publicações individuais $O$ girassol e a lua (200o), Meu coração, não sei por quê (2001), CRÍTICA! (2004), Mesa para dois (2006) e FANZINE (2007), que inicia - ainda limitada ao campo das HQs brasileiro, mas ali de forma marcante - a linha "autoral" na obra de Moon e Bá, ${ }^{4}$ que mais tarde encontrou continuidade e canonização no campo internacional. Nesse contexto, os gêmeos sublinham a natureza "literária" de sua obra, posicionando-se explicitamente enquanto "escritores" e sublinhando a importância da narrativa, da escrita em um romance gráfico:

If I write books, I'm a writer. If I make comics, I'm a cartoonist, but it's quite common for people to refer to me as an artist. Well, yes, but I'm a writer, too. Words are everything in a novel, and the script is everything in a good comic book. Yes, the drawings will draw the attention of the reader's eyes and carry the magic of translating the world into strokes and lines, and many cartoonists become cartoonists because they like to draw, but the images in a comic book are there to help tell a story. The story is more important. After some pages, the reader dives into the story and doesn't pay attention to the art anymore, because good art is invisible. And the story is a lot more than just the words written inside the captions and the balloons - more than narration and dialogue. The story is the union between words and pictures that was built in the script (BÁ; Moon, 27 de outubro de 2015).

3 Para o contexto dos EUA, é altamente simbólica a intervenção de Hillary Chute na revista da MLA: "But comics presents problems we're still figuring out (the term doesn't settle comfortably into our grammar; nomenclature remains tricky and open to debate). The field hasn't yet grasped its object or properly posed its project. To explore today's comics we need to go beyond preestablished rubrics: we have to reexamine the categories of fiction, narrative, and historicity" (Chute, 2008, p. 452)

4 Os títulos da série foram publicados por editoras do campo "independente", caraterizado também no Brasil pelo polo estético por obra "de autor". Há muito tempo estão fora de catálogo, e em 2017 Moon e Bá optaram pela sua reedição exclusivamente digital no portal Social Comics (https: //www.socialcomics.com.br/), com um modelo de streaming com assinatura mensal. 


\section{HQ e a literatura brasileira}

A discussão em torno da "literaricidade" ou não da sua obra e das HQs em geral leva inevitavelmente a questões dos contextos de produção, circulação e recepção no respectivo campo. Com isso, a obra dos gêmeos complica a situação, porque desde há mais de uma década se situa em ou entre vários campos, não somente entre o "puramente literário" e o "cultural" mais amplo, mas também entre o campo brasileiro, o norte-americano e, a partir desse último, o campo internacional. Dali surge uma dinâmica produtiva entre o Brasil e os Estados Unidos e os seus campos de produção e distribuição de HQs, atravessada pela diferenciação entre a produção "clássica" de HQ e a sua vertente mais "literária":

F[ábio] M[oon]: In Brazil, even if we're published by a publisher, we still do a lot of self-promotion, we sell a lot of books ourselves, we make a lot of effort to make people look at comics as a respectable medium, and we self-publish our work if we have to because we believe in it and we have learned that sometimes it's better to be self-published because not every project fits a certain publisher and the freedom and speed involved in making a self-published comic is always very tempting (FERNÁnDEZ, 2010).

Com isso, podemos continuar, ainda que como possibilidade, considerando a obra dos gêmeos formando parte das HQs e da literatura brasileira. A carreira deles se desenvolve ao mesmo tempo que as transformações no campo literário brasileiro evidenciam o crescente reconhecimento das HQs no campo cultural em geral. Mais ainda, Moon e Bá fazem parte da geração de autores e desenhistas que contribuiu para o estabelecimento do gênero logo após as transformações ocorridas nos anos 1990 no campo brasileiro, como explica Roberto dos Santos:

[L]evar obras de qualidade a um público-leitor ávido por novidades (nacionais ou estrangeiras) e que pode arcar com preços mais altos. [...] A ousadia permitiu que chegassem às prateleiras obras realizadas por artistas brasileiros que refletem a maturidade artística, técnica, narrativa e temática alcançada pelo quadrinho nacional (SANTOS, 2001, p. 10).

Numa entrevista mais recente, não por acaso publicada numa revista "literária" dos Estados Unidos, Gabriel Bá e Fábio Moon confirmam esta visão a partir da sua própria perspectiva, sublinhando a falta de uma tradição própria no campo das HQs no Brasil, o que fez com que a tradição norte-americana, internacionalmente estabelecida na segunda metade do século XX, servisse de modelo e motor para as transformações ocorridas no Brasil em torno da passagem do milênio:

GB: Only very recently are Brazilian authors looking for longer, more serious, and deeper narratives, which can be called graphic novels. The real truth is, despite the fact that comics started to be produced at the 
end of nineteenth century, we still don't have a graphic novel tradition, we don't have any classical works produced more than ten or fifteen years ago. Maybe the oldest and most relevant is Diomedes, A trilogia do acidente, by Lourenço Mutarelli, published between 1999 and 2002. FM: This movement happened after Lourenço Mutarelli published his first novels ( $O$ cheiro do ralo and Natimorto), after Rafael Coutinho published his graphic novel Cachalote, together with the writer Daniel Galera, and after Bá and I won the Jabuti Award with our adaption of O Alienista by Machado de Assis (Lino, 2018).

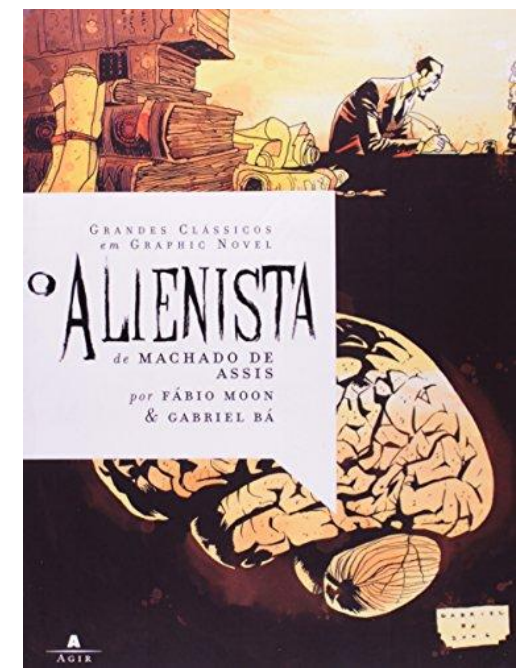

Quando O Alienista, na adaptação de Moon e Bá, foi premiado com um Jabuti em 2008 , foi ainda na categoria "Didático ou paradidático do ensino fundamental e médio". Portanto, o reconhecimento e, com ele, o passo à frente no estabelecimento das HQs no campo literário brasileiro, viveu certa limitação ou relativização através da suposta "utilidade didática" do gênero. Somente a partir de 2017, é que passou a existir a categoria "história em quadrinhos" no Prêmio Jabuti. Este contraste através de um período de nove anos ilustra bem a profundidade e a magnitude da evolução e da diversificação que o campo das HQs no Brasil vem experimentando neste início do século XXI. Mais uma vez é Waldomiro Vergueiro quem melhor a sintetiza:

[A] expansão maior do mercado de quadrinhos no Brasil ocorreu no segmento destinado ao público adulto. Vários autores que produziam regularmente para esse mercado e que apenas trafegavam pelos caminhos da produção alternativa conseguiram ter seus trabalhos publicados por empresas comerciais, o que representou grande passo para a área. As últimas duas décadas apenas intensificaram a tendência de lançamentos de quadrinhos para públicos diferenciados, acompanhando um processo de segmentação de mercados também ocorrido em outros países (VERgueiro, 2017, pos. 1597). 
Também se intensificam as interações com outros campos de produção cultural, entre eles o da literatura no sentido "clássico", como os exemplos mencionados acima. Aqui entra uma subdivisão interna muito interessante no campo da HQ, apontada por Havane Bezerra de Melo na sua dissertação de mestrado: a diferenciação e o conflito entre "história em quadrinhos - comics" e "romance gráfico - graphic novel" enquanto subgênero mais prestigiado e respeitado em termos dos valores da literatura tradicional (Melo, 2015, p. 9). Na terminologia de Bourdieu, esta polaridade espelharia a oposição entre o polo comercial e do consumo de massas e o polo estético, elitista. A discussão mostra que as delimitações não são claramente definidas, mas que a diferenciação existe e tem seus efeitos:

A mudança ocorreu, principalmente, em decorrência da entrada no mercado nacional do formato graphic novel, que já vinha sendo trabalhado no exterior há algumas décadas; porém, apenas por volta dos anos 2000 é que tivemos aumento de produção e popularização de obras nacionais. Assim, o termo graphic novel ganhou sua versão no idioma nacional e hoje é conhecido como "romance gráfico" ou "novela gráfica" (MELO, 2015, p. 9).

O gênero vem ganhando presença e prestígio no campo brasileiro, incluindo a importação de fora (principalmente dos Estados Unidos) e a produção "interna" por autores e desenhistas brasileiros. Constata-se a mesma lógica e contradição que o gênero evidencia em outros países, combinando a elevada ambição estética e qualidade artística do romance gráfico com o grande sucesso comercial dos "livros" de maiores custos de produção e venda, confirmando no século XXI o surgimento de um público mais exigente, em termos estéticos, e ao mesmo tempo mais solvente, em termos econômicos:

Nos últimos anos, até mesmo, a opção pela publicação de álbuns e graphic novels para um público com maiores condições financeiras se tornou mais comum entre alguns editores brasileiros, acompanhando a tendência mundial. [...] Muitos autores brasileiros também optaram pelo álbum ou pela graphic novel como o formato preferencial para seu trabalho artístico, com exclusão de outras possibilidades (VErgueiro, 2017, pos. 1619).

A discussão mostra que as delimitações não são claramente definidas, mas que a diferenciação existe e tem seus efeitos. Um desses efeitos é justamente uma aproximação ainda maior entre as HQs e o âmbito "do literário" em sentido tradicional, não somente na percepção crítica e geral do gênero, mas também - e este é o ponto decisivo - nas formas de recepção (por um público "leitor de literatura"), distribuição (cada vez mais presente em livrarias do que somente em "gibitecas") e produção (seja com editoras "literárias" entrando no mercado das HQs, como a Companhia das Letras com o selo "Quadrinhos na Cia.", seja a respeito das técnicas narrativas empregadas pelos autores). Juliane Blank formula esta contradição muito produtiva de forma sucinta: 
O conceito de "graphic novel" foi criado em primeiro lugar para o marketing e até hoje é utilizado, neste sentido, de forma excessiva, entretanto, de certa forma fez surgir também uma evolução da HQ numa direção que sim parece ter influências de métodos da narrativa e da produção literárias (BLANK 2014, p. 34, trad. minha). ${ }^{5}$

\section{HQ e a literatura internacionais}

Pascale Casanova, outra representante central da sociologia da literatura, no seu estudo já clássico La République Mondiale des Lettres, contempla a diferenciação entre os campos nacional e internacional na produção e circulação do literário, um aspecto que se aplica também à lógica no campo das HQs:

La position de chaque espace national dans la structure mondiale dépend de sa proximité à l'un des deux pôles, c'est-à-dire de son volume de capital. On peut donc se représenter l'univers littéraire mondial comme un ensemble formé des champs littéraires nationaux, eux-mêmes bipolarisés et situés différentiellement (et hiérarchiquemendants) la structure mondiale selon le poids relatif qu'y détiennent le pôle international et le pôle national (et nationaliste). La distribution inégale du capital littéraire dans l'univers littéraire se double d'une distribution inégale de capital linguistico-littéraire (CASANOVA, 2002, p. 8).

Enquanto autores brasileiros, Bá e Moon enfrentam o desequilíbrio que segundo Casanova marca o campo literário internacional, com a sua diferenciação hierarquizante entre literaturas "centrais" e "marginais", e com a sua seleção elitista do que faria parte da "literatura mundial". Sofrem, em princípio, das mesmas dificuldades de conseguir serem publicados e reconhecidos nos "centros" do campo internacional. Entretanto, o campo das HQs apresenta uma constituição e lógica bem diferente a esse respeito, pois parece mais permeável para os atores individuais - nesse caso os autores - passarem de um campo para outro. Bá e Moon, por exemplo, desde há quase duas décadas vem produzindo e sendo publicados em dois campos diferentes, o brasileiro e o norte-americano, e a partir deste, ainda no campo internacional.

Desde que passaram a atuar também no campo norte-americano, a partir do início do século XXI, o modo de produção e publicação, circulação e recepção da obra de Moon e Bá nos obriga a questionar as categorizações tradicionais e inequívocas, como nos lembra Piter Costa:

Daytripper, de Moon e Bá, autores brasileiros que também trabalham no mercado americano, foi publicada originalmente em inglês, justamente

5 Original: "Der Begriff Graphic Novel wurde zwar in erster Linie als Marketing-Begriff eingeführt und wird in diesem Sinne bis heute inflationär verwendet, aber es gibt auch eine gewissermaßen nachfolgende Entwicklung des Comics in eine Richtung, die durchaus von Verfahren literarischen Erzählens und von literarischen Produktionsmethoden beeinflusst scheint”. 
para esse mercado. Os autores são brasileiros, o cenário da história é brasileiro, a produção se deu no Brasil, mas a publicação foi nos EUA, em inglês. Daytripper compõe o Opus de Moon e Bá. Mas se levarmos em conta alguns fatores como tempo, local, língua e editora inicial da publicação, não diríamos que faz parte do Thesaurus brasileiro (CosTA, 2016, p. 84-85).

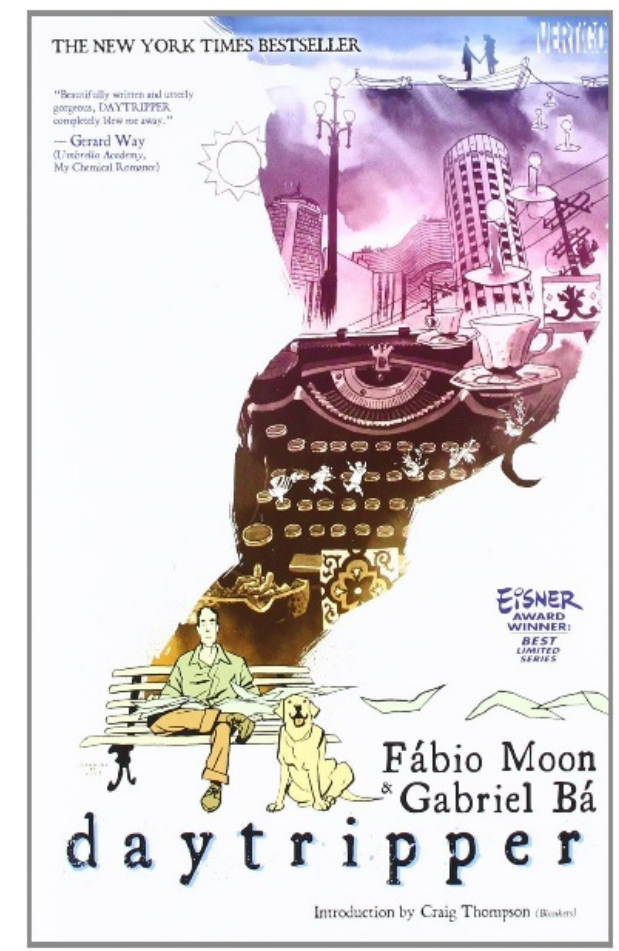

Um detalhe importante que complica esta categorização é que Daytripper foi escrito e publicado originalmente em inglês (pelos próprios Moon e Bá) e mais tarde traduzido (por Érico Assis) para a edição brasileira. Neste momento, eles parecem eliminar os traços da sua condição de "autores brasileiros". Porém, há também indícios na direção contrária, reestabelecendo a identidade brasileira dos gêmeos, por exemplo o lema comercial criado em volta deles e apropriado pelos dois para a sua auto-imagem enquanto "Brazil's very own wonder twins"; ou a apresentação de Daytripper da editora Panini no Brasil ${ }^{6}$ como "a primeira produção $100 \%$ verde e amarela da linha nos Estados Unidos”. Da mesma forma, situar as tramas no Brasil, inclusive nas produções estadunidenses, reforça esta dinâmica, como confirma Bá numa entrevista, referindo-se à dimensão da substancialidade das histórias a partir da realidade brasileira:

GB: Part of Brazilian society, its culture and manners were told, constructed by literary books more so then history ones. The way we see ourselves

\footnotetext{
6 https:/hotsitepanini.com.br/vertigo/daytripper-de-fabio-moon-e-gabriel-ba-em-2o11/
} 
and relate to each other is always a comparison to archetype characters of great masterpieces of our literature. [...] We need to be true to ourselves - thus placing the story in Brazil - in order to achieve a level of honesty with the story that will really touch the reader (FERNÁNDEZ, 2010). ${ }^{7}$

THE NEW YORK TIMES BESTSELLE

Fábio Moon É Gabriel Bá

daytripper

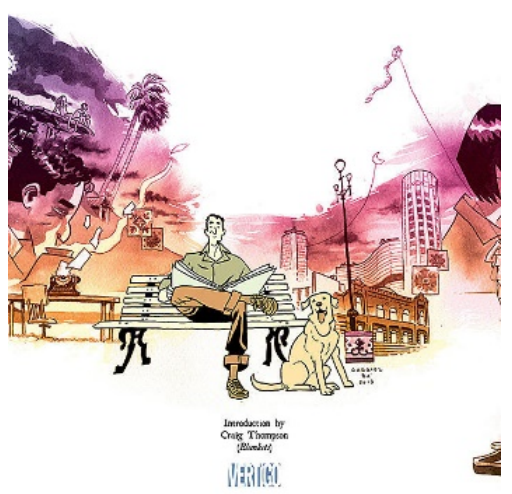

Seguindo esses movimentos e gestos, o exemplo de Moon e Bá serve muito bem para retraçar os posicionamentos estratégicos enquanto autores de HQ "brasileiros" e "internacionais", acumulando capital simbólico a partir dos Estados Unidos e repassando entre os dois países e campos culturais. Uma marca nessa trajetória é, sem dúvida, o volume De: tales que contém uma série de histórias curtas ou "contos", antes publicados em português de forma individual e ali reunidos em tradução para o inglês publicada nos Estados Unidos, como explica David William Foster:

De:tales. Stories from urban Brazil was published by Dark Horse Books in 2006: quite a milestone for Latin American graphic art, since the most famous graphic artist in Spanish, Quino (Salvador Joaquín Lavado), creator of Mafalda (1966-73), which has been translated into dozens of languages, has yet to be available in English (Foster, 2008, p. 2).

7 De forma análoga, David William Foster "detecta" a presença e a influência da cidade de São Paulo no volume De:tales. "This setting is never explicitly identified as São Paulo, although to be sure, it is evidently so as a combined consequence of the identity of the authors, internal details resembling the physical appearance of that Brazilian city, and the way in which the language of signage and such is in Portuguese, being left untranslated into English. [...] The result is that the characters of the strips -recurrently Moon and Bá in the guise of their artistic alter egos - are very much the Everyman (and Everywoman) of urban (post)modernity" (FosTER, 2008, p. 4). 


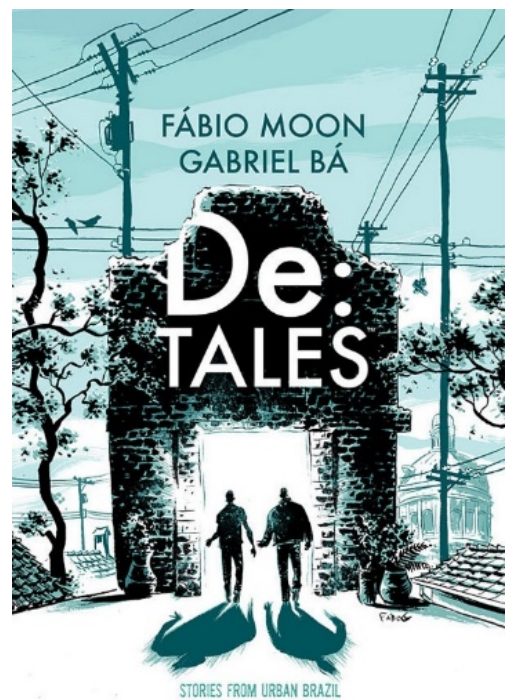

Desde então, a presença e acumulação de capital simbólico dos gêmeos no campo das HQs estadunidense foi aumentando e chegou a um clímax em 2010 com Day Tripper, obra de grande sucesso e prestígio internacional. Na adaptação do romance Dois irmãos (2015), de Milton Hatoum, se pode então, verificar a reversão desse capital simbólico acumulado internacionalmente, num cruzamento de gêneros e territórios dentro do campo literário brasileiro (cf. ToмÉ, 2013).

Ao mesmo tempo, eles atuam dentro dessa tensão e polaridade, no polo comercial das HQs clássicas, participando enquanto desenhistas em produções como Hellboy, de Mike Mignola, Umbrella academy, de Gerard Way, ou Casanova, de Matt Fraction, e com obras autorais no polo estético, além das já mencionadas (Daytripper, O Alienista, Dois irmãos) mais recentemente com a adaptação de maior prestígio internacional, How to talk to girls at parties, do conto do mesmo título de Neil Gaiman. ${ }^{8}$ Assim, Moon e Bá participam ativamente em duas dinâmicas diferentes de acumulação de capital simbólico dentro dos campos literário e culturais envolvidos. A essa dupla dinâmica correspondem dois sistemas de circulação das obras e autores, dois mercados literários: os Estados Unidos enquanto "pátria" do romance gráfico e o Brasil enquanto mercado globalmente marginal (ou marginalizado) e ainda fortemente marcado pela tradição europeia. A passagem do Brasil e da língua portuguesa para os Estados Unidos e a língua inglesa implica uma dimensão política, estratégica, como bem mostra Foster:

[...] this strategic linguistic decision [...] has very much to do with the rise of the graphic novel as a major cultural genre in English and with the

\footnotetext{
8 Cf., nesse contexto, as seguintes publicações: Mignola; BÁ; Moon; Corben, 2016, e Mignola; BÁ; MoOn, 2019; WAY; BÁ, 2008, 2009 e 2019; Fraction; BÁ; MoOn, 2014 e 2015; FraCtion; BÁ, 2015; Fraction; BÁ; Moon; Chabon, 2015 e 2017, assim como Gaiman; BÁ; Moon, 2016.
} 
fact that, in Brazil, there is an association of the cultural vanguard with the English language (FosTER, 2016, pos. 1784).

Particularmente o maior sucesso de autoria própria e de prestígio estético dos gêmeos, Daytripper, parece conter essa dupla posição, adequando-se às tradições vigentes nos dois mercados, combinando as suas respectivas lógicas e superando as contradições nisso implicadas:

Daytripper foi pensado para funcionar de duas maneiras. Era uma história que tinha que funcionar no mercado americano, tinha que pensar no público americano e no jeito que eles publicam quadrinho. Então tinha que funcionar em capítulos do jeito que o leitor quisesse voltar todos os meses. A gente tinha que pensar em capítulos que funcionassem bem separadamente. E, depois, o conjunto tinha que funcionar também, porque a gente sabia que, pelo menos no Brasil, ia sair de uma vez só, como um livro (Melo, 2015, p. 97).

Ao mesmo tempo, no circuito internacional Moon e Bá introduzem temáticas, estilos e escritas relacionadas ao Brasil, enquanto contribuem com produções brasileiras para uma maior projeção internacional do Brasil no campo global das HQs e, possivelmente, também da literatura. É notável essa lógica de agir como "autores brasileiros" sem que este fato seja necessariamente percebido na circulação e recepção global. Já vimos algumas das referências intencionadas à origem brasileira dos gêmeos, também as menções da atual geração brasileira de autores de HQ pelos dois, sempre no sentido de ligar esse detalhe com o circuito norte-americano, onde se integraria na lógica internacional, como sugere também Foster:

[...] These narratives show little interest in dealing with the sociopolitical issues of dictatorship and redemocratization in Brazil. Rather, they are very much tied to the aggressive culture of modernity that has characterized Brazil in recent decades, a culture that is ever attuned to matters of internationalism, globalization, and cross-cultural identity, especially where English and American life are involved (Foster, 2016, pos. 115).

Um "detalhe" que vale a pena considerar é o da língua original das obras publicadas pelos gêmeos. Depois da mudança para os Estados Unidos, começam a escrever a parte textual de suas obras diretamente em inglês, fato que é tematizado em algumas entrevistas, mas sempre de forma anedótica, sem questionar esse fato em termos de capacidade linguística para o nível necessário na produção literária. No caso da literatura tradicional, o fato de um autor escrever em outra língua da língua materna/primeira ou segunda dele é extremamente raro e só se legitima através de biografias migratórias e concepções estéticas da hibridação, que vão orientar completamente a leitura e análise dessas obras pela crítica e academia. ${ }^{9}$

9 Entre os exemplos de maior projeção internacional se destacam Yoko Tawada, escritora japonesa radicada na Alemanha desde os anos 1980 que escreve quase a totalidade da sua obra em alemão; 
Outro exemplo interessante é a adaptação de Moon e Bá do romance Dois irmãos, de Milton Hatoum, publicada primeiro no Brasil em 2015, e somente depois (ainda que no mesmo ano, enquanto "translated from its Brazilian edition", mas sem indicação de tradutor/a) nos Estados Unidos. Assim, a ordem cronológica parece voltar ao "normal". Ao mesmo tempo, o impulso inicial saiu da editora brasileira, que contratou os artistas internacionalmente estabelecidos, para darem nova versão e visibilidade a um romance (de 200o) que hoje figura entre os clássicos da literatura brasileira contemporânea.

A apresentação e recepção desse livro já não tem nada em comum com o que se observava em torno a O Alienista, oito anos antes.

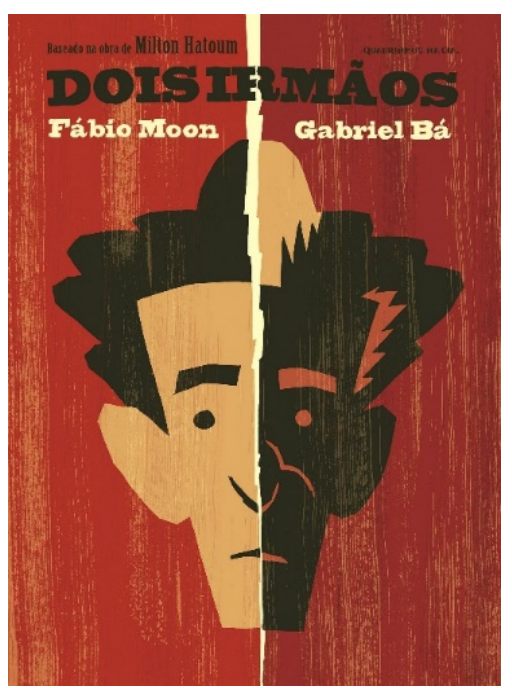

Parece mesmo que essa publicação se rege por uma lógica dupla: num primeiro momento é o gênero mais popular do "romance gráfico" que ajuda a divulgar (de novo ou de outra forma) uma obra do gênero central no campo literário - o romance. Mas também é o romance canonizado que aqui "transfere" algo de seu capital simbólico para o seu "parente" no (sub)campo das HQs, mas sem continuar, por isso, a relação hierárquica tradicional entre "original" e "adaptação", reproduzindo melhor a nova lógica das atuais adaptações transgenéricas enquanto releitura e variação na cultura popular. ${ }^{10}$

Comics adaptations of literary texts are closely linked to canonization processes. Serial graphic narratives about 'world literature classics' as

Aki Shimazaki, uma japonesa que também desde os anos de 1980 vive no Canadá e escreve os seus romances em francês, e também escritores clássicos como Vladimir Nabokov e Joseph Conrad, ambos emigrantes, da Rússia para os EUA e da Polônia para a Inglaterra, respectivamente, que se tornaram famosos como escritores de língua inglesa.

10 Lucas Piter Costa analisa o lançamento da obra com a participação de Milton Hatoum no Salon du Livre 2015 como confirmação dessa subversão das hierarquias tradicionais, reduzindo Hatoum à função de "agente legitimador de Moon e Bá" e confirmando o caráter do evento enquanto "cena de enunciação quadrinística” (CosTA, 2016, p. 172). 
well as single graphic novel releases adapting literary texts prefer canonized works as their starting platforms. In a way, comic adaptations prove the status of a text as a canonized work and, at the same time, confirm this status (Schmitz-EmANs, 2013, p. 397).

De volta no campo internacional, em 2016 a adaptação do conto de Neil Gaiman, How to talk to girls at parties, publicado originalmente em 2006 na coletânea Fragile thing: short fiction and wonders, confirma este modelo, justamente com a obra literária de um autor que conquistou a fama internacional enquanto autor da série de romances gráficos Sandman entre 1989 e 1996 e depois enquanto escritor "clássico" cujos romances e contos vem sendo adaptados às HQ por diferentes desenhistas. ${ }^{11}$

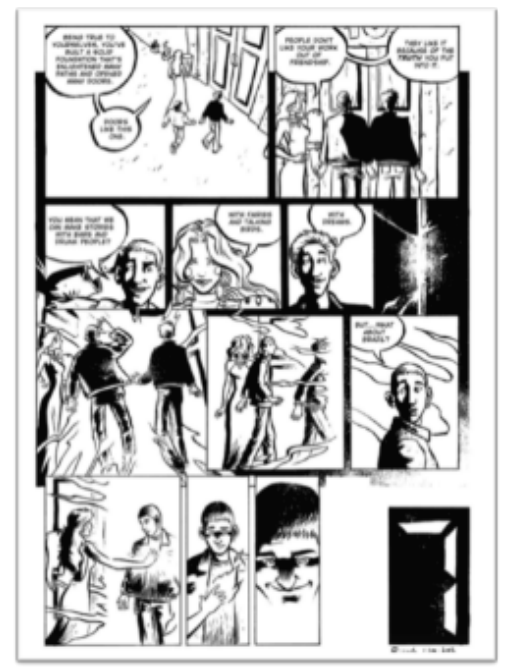

\section{Considerações finais}

A história de abertura do volume De: tales (que não leva título ou faz parte do "Prefácio") representa, numa sequência onírica, a busca dos gêmeos por uma forma própria e adequada de expressão artística. No momento final do sonho, eles perguntam à fada Ursula e ao pássaro Pip qual seria o lugar do Brasil no seu projeto de contar histórias sobre "bares e bêbados, sobre fadas e pássaros falantes, sobre sonhos..." em vez de uma resposta, a fada põe a mão sobre o coração de um dos gêmeos. Este gesto carregado de simbolismo, realizado de forma consciente no início da primeira publicação de significativo alcance internacional, articula a volta à pergunta se Fábio Moon e Gabriel Bá ainda podem ser considerados parte da "literatura brasileira". Revendo a fortuna crítica existente, e se aceitarmos a inclusão das HQs e da narrativa gráfica contemporânea no campo literário, até

\footnotetext{
"Gaiman é de fato o exemplo ideal do jogo das passagens entre vários meios e gêneros, como no exemplo mais conhecido e recente de American gods (primeiro em romance em 2001, depois seriado de TV em 2017, e desde 2018 também como romance gráfico).
} 
as suas obras mais recentes, originalmente publicadas em inglês e nos Estados Unidos, podem ser vistas como elementos da literatura brasileira:

With Daytripper, Fábio Moon and Gabriel Bá demonstrate not only that they are capable of uniquely original narratives, but that the overall quality of this title makes it an important entry in the bibliography of contemporary Brazilian fiction and a fine example of the level of the graphic narrative attained in Brazilian culture (FosTER, 2016, pos. 1938).

Entretanto, também ficou claro ao longo deste percurso pela obra multifacetada de "Brazil's very own wonder twins" que a questão exclusivamente "literária" não é suficiente para abranger as dimensões e significados que esta obra exemplifica e implica. Fica também evidente que o olhar desde o âmbito tradicionalmente "literário" já não tem fundamento para o desprezo ou a hierarquização desde cima. As HQs, hoje em dia, exercem igual ou até mais influência cultural, têm um papel simbólico e social mais relevante e importante do que a literatura em sentido clássico.

Le principe du changement des oeuvres réside dans le champ de production culturelle et, plus précisément, dans les luttes entre des agents et des institutions dont les stratégies dépendent de l'intérêt qui'ils ont, en fonction de la position qu'ils occupent dans la distribution du capital spécifique (institutionnalisé ou non), à conserver ou à transformer la structure de cette distribution, donc à perpétuer les conventions en vigueur ou à les subvertir [...] (Bourdieu, 1992, p. 325).

Ao contrário, é necessário abrir a visão e ampliar o enfoque analítico, porque hoje em dia as representações da cultura pop(ular) contemporânea, possivelmente, têm mais influência e relevância social (e até política) do que a literatura em sentido tradicional, cada vez mais marginalizada e presa numa contradição entre a massificação (e consequentemente a perda de profundidade e simbolismo) e o isolamento elitista (a famosa "torre de marfim" da "alta literatura"). Algumas formas da literatura tradicional estão saindo do grande contexto, explorando e ocupando, às vezes até construindo novos (sub)campos, como pode ser observado, por exemplo, na volta da poesia em estreita ligação com a cultura pop, com a música em geral e o rap em particular; no princípio do do-it-yourself (diy) na publicação e distribuição de textos, misturando modelos estéticos da gama inteira, do clássico ao punk; na volta da oralidade nos poetry slams, enfocando o evento ao vivo, a performance do texto etc.

Por isso, na sua relação multifacetada com a literatura, as HQs talvez sejam uma forma de expressão artística hoje em dia mais presente e mais relevante do que a literatura tradicional. Mais ainda, o próprio status da "cultura alta/erudita" deve ser questionado hoje, frente às profundas transformações da chamada "cultura de massa”, em cada vez mais casos transformada em portadora de simbolismos e significados culturais de forte impacto e grande influência. Essa dinâmica 
complexa inclui as novas formas de produção e consumo audiovisual (cinema, séries, música). As HQs participam dos novos diálogos e interações entre as artes e os gêneros, como por exemplo as adaptações de HQ para o cinema e a TV, mas também como parte das interações e hibridações globais entre as diferentes tradições de HQ (mangá japonês e asiático, HQ ou banda desenhada europeia, graphic novel estadunidense).

Como consequência, no interior do campo das HQs estão acontecendo variados processos de diferenciação, estruturação e canonização, estão se estabelecendo os clássicos do campo e dos gêneros nele contidos, estão se formando linhas de uma tradição própria, ${ }^{12}$ e com isso o campo também está se reaproximando do funcionamento da literatura no sentido tradicional.

\section{Referências}

BÁ, Gabriel; Moon, Fábio. O girassol e a lua. São Paulo: Via Lettera, 2000.

BÁ, Gabriel; Moon, Fábio. Meu coração, não sei por quê. São Paulo: Via Lettera, 2001.

BÁ, Gabriel; Moon, Fábio. CRÍTICA! São Paulo: Devir, 2004.

BÁ, Gabriel; Moon, Fábio. Mesa para dois. São Paulo: Devir, 2006.

BÁ, Gabriel; Moon, Fábio. De:tales. Stories from Urban Brazil. Milwaukie; London: Dark Horse; Diamond, 2006.

BÁ, Gabriel; Moon, Fábio. Fanzine. São Paulo: Devir, 2007.

BÁ, Gabriel; Moon, Fábio. Daytripper. Burbank: DC Comics, 2011.

BÁ, Gabriel; Moon, Fábio. Dois irmãos. Baseado na obra de Milton Hatoum. São Paulo: Quadrinhos na Cia, 2015.

BÁ, Gabriel; Moon, Fábio. Bá and Moon on the Making of Two Brothers, Part I: introduction. Comics Alliance, 26/10/2015. Disponível em: https://comicsalliance. com/ba-moon-making-of-two-brothers-introduction/. Acesso em: 25 mar. 2019.

BÁ, Gabriel; Moon, Fábio. Bá and Moon on the Making of Two Brothers, Part II: the story of a script. Comics Alliance, 27/10/2015. Disponível em: http://comicsalliance. com/ba-moon-making-of-two-brothers-script/. Acesso em: 25 mar. 2019.

Blank, Juliane. Vom Sinn und Unsinn des Begriffs Graphic Novel. Berlin: Ch. A. Bachmann, 2014.

\footnotetext{
${ }_{12}$ Lembrando aqui, no final do texto e ao pé da página, as palavras de Antonio Candido na Formação da literatura brasileira, onde apresenta o conceito da "literatura como sistema": "As obras não podem aparecer em si, na autonomia que manifestam, quando abstraímos as circunstâncias enumeradas; aparecem por força da perspectiva escolhida, integrando em dado momento um sistema articulado e, ao influir sobre a elaboração de outras, formando, no tempo, uma tradição" CANDIDO, 2000, p. 24).
} 
BouRdieu, Pierre. Les règles de l'art. Genèse et structure du champ littéraire. Paris: Editions du Seuil, 1992.

Bourdieu, Pierre. Une révolution conservatrice dans l'édition. Actes de la recherche en sciences sociales (126-127), p. 3-28, 1999. Disponível em: www.persee.fr/doc/ arss_0335-5322_1999_num_126_1_3278. Acesso em: 25 mar. 2019.

CANDido, Antonio. Formação da literatura brasileira: momentos decisivos. $6^{\mathrm{a}}$. ed. Belo Horizonte: Editora Itatiaia, 2000.

Casanova, Pascale. Consécration et accumulation de capital littéraire. La traduction comme échange inégal. Actes de la recherche en sciences sociales (144), p. 4-20, 2002. Disponível em: http://www.persee.fr/doc/arss_0335-5322_2002_num_144_ 1_280404. Acesso em: 25 mar. 2019.

Chute, Hillary. Comics as Literature? Reading Graphic Narrative. PMLA, vol. 123, no. $2,2008$.

Costa, Lucas Piter Alves. Uma análise do discurso quadrinístico: práticas institucionais e interdiscurso. 2016. Tese de Doutorado - UFMG, Faculdade de Letras, Belo Horizonte, 2016.

Etter, Lukas; Stein, Daniel. Comictheorie(n) und Forschungspositionen. In: Abel, Julia; Klein, Christian (orgs.). Comics und Graphic Novels. Eine Einführung. Stuttgart: J.B. Metzler, 2016.

Fernández, David. Zona Negativa interviews Fábio Moon \& Gabriel Bá. Zona Negativa, 2010. Disponível em: https://www.zonanegativa.com/zona-negativainterviews-fabio-moon-gabriel-ba/. Acesso em: 25 mar. 2019.

Foster, David William. Drawing São Paulo: the graphic fiction of Fábio Moon and Gabiel Bá. CiberLetras, 19, 20o8. Disponível em: http://www.lehman.cuny. edu/ciberletras/v19/foster.html. Acesso em: 25 mar. 2019.

Foster, David William. El Eternauta, Daytripper, and beyond. Graphic narrative in Argentina and Brazil. Austin: University of Texas Press, 2016.

Fraction, Mike; BÁ, Gabriel. Casanova: Vol. 3: Avaritia - The Complete Edition. Portland: Image Comics, 2015.

Fraction, Mike; BÁ, Gabriel; Moon, Fábio. Casanova: Vol. 1: Luxuria - The Complete Edition. Portland: Image Comics, 2014.

Fraction, Mike; BÁ, Gabriel; Moon, Fábio. Casanova: Vol. 2: Gula - The Complete Edition. Portland: Image Comics, 2015.

Fraction, Mike; BÁ, Gabriel; Moon, Fábio; Chabon, Michael: Casanova: Acedia, Vol. 1. Portland: Image Comics, 2015.

Fraction, Mike; BÁ, Gabriel; Moon, Fábio; Chabon, Michael. Casanova: Acedia, Vol. 2. Portland: Image Comics, 2017. 
Gaiman, Neil; BÁ, Gabriel; Moon, Fábio. How to talk to girls at parties. London: Headline, 2016.

GarcíA, Santiago. On the graphic novel. Jackson: University Press of Mississippi, 2015 .

KaINDL, Klaus. Das Feld als Kampfplatz - Comics und ihre Übersetzung im deutschen Sprachraum. In: BAChleitner, Norbert; Wolf, Michaela (orgs.). Streifzüge im translatorischen Feld. Zur Soziologie der literarischen Übersetzung im deutschsprachigen Raum. Münster: LIT, 2010, p. 331-350.

Lino, Patricia.: Two Brazilian Graphic Novelists: Gabriel Bá and Fábio Moon: A Conversation with Patrícia Lino. Latin American Literature Today, 6, 2018. Disponível em: http://www.latinamericanliteraturetoday.org/en/2018/may/ two-brazilian-graphic-novelists-gabriel-b\% $\mathrm{C}_{3} \% \mathrm{~A}_{1}$-and-f\% $\mathrm{C}_{3} \%$ A1bio-moonconversation-patr\% $\mathrm{C}_{3} \%$ ADcia-lino. Acesso em: 25 mar. 2019.

Melo, Havane Maria Bezerra de. Romance gráfico brasileiro. A construção de um gênero quadro a quadro. 2015. Dissertação de Mestrado - Programa de PósGraduação em Comunicação, Universidade de Brasília, Brasília, 2015.

Mignola, Mike; BÁ, Gabriel; MOON, Fábio. B.P.R.D.: Vampire. Milwaukie: Dark Horse, 2019.

Mignola, Mike; BÁ, Gabriel; MOON, Fábio ; Corben, Richard; Mahon, Mick. Hellboy in Mexico. Milwaukie: Dark Horse, 2016.

Schmitz-Emans, Monika. Graphic Narrative as World Literature. In: Stein, Daniel (org.). From comic strips to graphic novels. Contributions to the theory and history of graphic narrative. Berlin: De Gruyter, 2013.

SÁnchez-MesA, Domingo; BAEtens, Jan. La literatura en expansión. Intermedialidad y transmedialidad en el cruce entre la Literatura Comparada, los Estudios Culturales y los New Media Studies. In: Tropelías, Zaragoza, 27, p. 6-27, 2017.

Santos, Roberto Elísio dos. O espaço do quadrinho brasileiro. Comunicação \& Inovação, vol. 1, no. 2, p. 8-12, 2001. Disponível em: http://seer.uscs.edu.br/index.php/ revista_comunicacao_inovacao/article/view/1303/955. Acesso em: 25 mar. 2019.

SiLva, Marie-Manuelle da. Banda desenhada literária ou literatura desenhada? In: RIBEIRO, Eunice (org.). Modernidades comparadas. Estudos literários/Estudos culturais revisitados. Braga: Húmus; Universidade do Minho, 2012.

TомÉ, Marcel Luiz. As inovações estéticas e narrativas nos quadrinhos autorais de Fábio Moon e Gabriel Bá: um estudo de Daytripper. Dissertação de Mestrado. 2013. Programa de Mestrado em Comunicação, Universidade Municipal de São Caetano do Sul, São Caetano do Sul, 2013.

Vergueiro, Waldomiro. Panorama das Histórias em Quadrinhos no Brasil. São Paulo: Peirópolis, 2017. 
Way, Gerard; BÁ, Gabriel. The Umbrella Academy. Apocalypse Suite. Milwaukie; London: Dark Horse; Diamond, 2008.

Way, Gerard; BÁ, Gabriel. The Umbrella Academy. Dallas. Milwaukie; London: Dark Horse; Diamond, 2009.

WAy, Gerard; BÁ, Gabriel. The Umbrella Academy. Hotel Oblivion. Milwaukie; London: Dark Horse; Diamond, 2019.

Recebido 31 de outubro de 2019.

Aprovado 4 de novembro de 2019.

\section{Resumo/Abstract/Resumen}

Entre vários campos: a obra de Fábio Moon e Gabriel Bá e a literatura brasileira

\section{Marcel Vejmelka}

A obra de Fábio Moon e Gabriel Bá evidencia de forma particular os conflitos e as tensões existentes entre os diferentes campos culturais envolvidos na produção e circulação de histórias em quadrinhos, inspirando a observar a sua evolução num jogo de forças e dinâmicas parcialmente conflitivas, aparentemente contraditórias, mas que afinal compõem um panorama concludente das caraterísticas do gênero e da sua circulação nos campos nacionais e internacionais.

Palavras-chave: HQ, narrativa gráfica, Gabriel Bá, Fábio Moon, literatura brasileira

Across various fields: the work of Fábio Moon and Gabriel Bá and Brazilian literature

The work of Fábio Moon and Gabriel Bá is particularly apt to show the conflicts and tensions that exist between the various cultural fields involved in the production and circulation of comics. It inspires us to observe their evolution, entangled by partially conflictive and apparently contradictory forces and dynamics, but which in the end offer a conclusive panorama of the genre and of their circulation at the national and international levels.

Keywords: comics, graphic narrative, Gabriel Bá, Fábio Moon, Brazilian literature

Entre varios campos: la obra de Fábio Moon y Gabriel Bá y la literatura brasileña

La obra de Fábio Moon y Gabriel Bá hace visibles, particularmente, los conflictos y las tensiones que existen entre los distintos campos culturales presentes en la producción y circulación del cómic, inspirándonos a observar su evolución, que 
es atravesada por fuerzas y dinámicas parcialmente conflictivas y aparentemente contradictorias, pero que acaban por componer un panorama concluyente de las características del género y de su circulación en el campo nacional e internacional.

Palabras clave: cómic, narrativa gráfica, Gabriel Bá, Fábio Moon, literatura brasileña. 